\title{
Design and Experimental Realization of a Broadband Transformation Media Field Rotator at Microwave Frequencies
}

\author{
Huanyang Chen, Bo Hou, Shuyu Chen, Xianyu Ao, Weijia Wen, and C. T. Chan* \\ Department of Physics, Hong Kong University of Science and Technology, Clear Water Bay, Kowlong, Hong Kong, China
}

(Received 24 January 2009; revised manuscript received 1 April 2009; published 8 May 2009)

\begin{abstract}
We designed a metamaterial field rotator that can rotate electromagnetic wave fronts. Our starting point was the transformation-media concept. Effective medium theories and full simulations facilitated the actual design process. We created at a very simple structure comprising of an array of identical aluminum metal plates. We made and measured a sample and we experimentally demonstrated the field rotation effect as well as the broadband functionality at microwave frequencies.
\end{abstract}

DOI: 10.1103/PhysRevLett.102.183903

PACS numbers: 42.25.Fx, 41.20.Jb, 42.25.Gy

Recently, the transformation-media concept has attracted the full attention of many researchers [1-26]. One reason for its popularity is its potential use in designs of invisibility cloaking devices [1-12]. Transformation media actually reaches beyond the ability to make things invisible. It can lead to many novel wave-manipulation devices, including field concentrators [13], field rotators [14,15], electromagnetic wormholes [16], impedance-matched hyperlenses [17], field shifters [18,19], anticloaks [20], cylindrical superlenses [21], superscatterers [22], superabsorbers [23], and remote cloaks [24]. Although the theory is flourishing, it is quite obvious that most real devices are very difficult to create in practice. It is useful to create devices for which real examples can be made and with which wave-manipulation functionality can be demonstrated experimentally. It is also desirable to create examples for which there are broadband functionalities, because the bandwidth issue is frequently identified as a major hurdle in the practical implementation of transformation-media devices. In this Letter, we describe an example with experimental evidence that demonstrates the functionality of a transformation-media based field rotator that operates at a wide bandwidth.

Based on the coordinate transformation methods [1,2], we proposed a two-dimensional transformation-media shell that can rotate fields but is itself invisible. The permittivity tensor and permeability of such a "field rotator" for transverse-electric (TE) polarized waves (whose magnetic field is in $z$ direction) [14] are, respectively, described as

$$
\stackrel{\leftrightarrow}{\varepsilon}=\left|\begin{array}{cc}
\varepsilon_{x x} & \varepsilon_{x y} \\
\varepsilon_{x y} & \varepsilon_{y y}
\end{array}\right|
$$

and $\mu_{z}=1$, where $\varepsilon_{x x}=\varepsilon_{u} \cos ^{2}(\theta+\tau / 2)+\varepsilon_{v} \sin ^{2}(\theta+$ $\tau / 2), \quad \varepsilon_{x y}=\left(\varepsilon_{u}-\varepsilon_{v}\right) \sin (\theta+\tau / 2) \cos (\theta+\tau / 2), \quad \varepsilon_{y y}=$ $\varepsilon_{u} \sin ^{2}(\theta+\tau / 2)+\varepsilon_{v} \cos ^{2}(\theta+\tau / 2)$, and $\varepsilon_{u}=\left(2+t^{2}-\right.$ $\left.t \sqrt{t^{2}+4}\right) / 2, \quad \varepsilon_{v}=\left(2+t^{2}+t \sqrt{t^{2}+4}\right) / 2, \cos \tau=\frac{t}{\sqrt{t^{2}+4}}$, $\sin \tau=\frac{2}{\sqrt{t^{2}+4}}$, where $t$ is a constant. If these conditions are satisfied, the shell itself is invisible while it performs the rotation. We note that for the ideal field rotator, $\varepsilon_{u}$ and $\varepsilon_{v}$ are related to $\tau$. However, such a condition can be relaxed. As long as $\varepsilon_{u}$ and $\varepsilon_{v}$ are different (anisotropic), the metamaterial shell can still function as a rotator, but the shell itself will cause some scattering. We focus on this "reduced" field rotator due to its easy implementation.

We start from the specific pattern shown in Fig. 1 and use rectangular metal plates as building blocks to produce the anisotropic metamaterial. We first break up a concentric cylindrical shell whose dimensions are $12.5 \mathrm{~mm}<$ $r<50 \mathrm{~mm}$ into six concentric shells of equal thickness $(6.25 \mathrm{~mm})$. We divide each shell into cells each measuring $6.25 \mathrm{~mm}$ in the middle. Each cell has "fanlike" shape as shown in Fig. 1(b). From the inside to the outside, the number of cells in each ring increases from 16 to 22 to 28 to 34 to 41 to 47 . A rectangular metal plate is placed in the

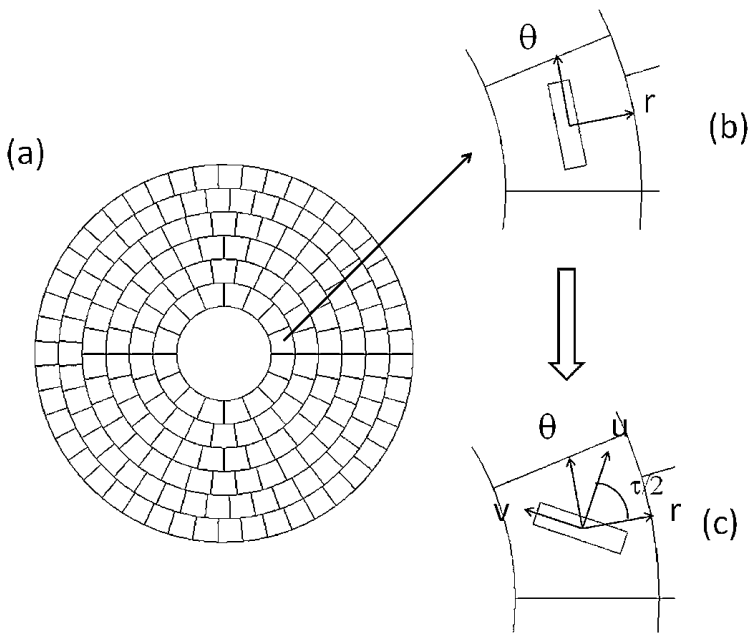

FIG. 1. (a) A division of the field rotator into many cells with "fanlike" shapes with their principal axes in the $r$ direction and the $\theta$ direction. (b) Each cell has a rectangular cylinder at its center with its longer side aligned with the tangential direction (the $\theta$ direction). (c) The field rotation effect can be achieved by rotating each rectangular cylinder around its center at a fixed angle $\tau / 2$. 
center of each cell so that the longer side is aligned with the tangential direction [the $\theta$ direction in Fig. 1(b)]. With a wavelength that is long compared with the metal plate dimensions, the metamaterial structure can be viewed as having concentric cylindrical shells with a positiondependent effective permittivity tensor, $\stackrel{\leftrightarrow}{\varepsilon}=\operatorname{diag}\left\{\varepsilon_{r}, \varepsilon_{\theta}\right\}$, and permeability $\mu_{z}$. This structure can also be viewed as a "zero-angle" field rotator, with parameters $\varepsilon_{u}=\varepsilon_{r}, \varepsilon_{v}=$ $\varepsilon_{\theta}, \mu_{z}$, and $\tau=0$ (a zero rotation angle). We note that $\mu_{z}$ is less than unity [27]. The above metamaterial device can be used to produce a field rotation effect simply by rotating each rectangle anticlockwise around its center by $\tau / 2$ [see Fig. 1(c)]. In the supplementary materials, we present the specific design with rectangles $\left(4 \times 1 \mathrm{~mm}^{2}\right)$ using the effective medium theory (EMT) [28]. The rotation angle depends on the anisotropy. The difference between $\varepsilon_{u}$ and $\varepsilon_{v}$ can be increased simply by using rectangles with larger aspect ratios. We use $\left(6 \times 1 \mathrm{~mm}^{2}\right)$ rectangles in the unit cell in the following section so that the rotation effect is stronger than the one in the supplementary materials. However, the EMT is not quantitatively accurate when the anisotropy is large, and in such situations, we have to employ brute-force full wave simulation for comparisons with experimental results. In the following, we set $\tau / 2=$ $\pi / 4$.

The experimental configurations are schematically shown in Fig. 2(a). The field rotator is a cylindrical object comprising 188 thin aluminum pieces arranged according to the theoretically designed pattern [see Fig. 2(b)]. We use a laser to cut the same pattern of slits with the same cross section in a thin slice of Plexiglas, and the $\mathrm{Al}$ pieces are inserted into these slits so that their relative positions can be fixed by the Plexiglas slice. By using several Plexiglas slices, we can fix the shape of the field rotator according to the theoretical blueprint, which has an annular cross section of $12.5 \mathrm{~mm}<r<50 \mathrm{~mm}$ and a length of $80 \mathrm{~cm}$.

In the experiment, the samples are placed horizontally with the axis orientated along the $z$ direction, and a hornshaped antenna at a distance of $\sim 90 \mathrm{~cm}$ away from field rotator transmits microwaves toward the samples with the $E$ field polarized along the vertical direction. To visualize the rotation effect of the electromagnetic field by the structure, we scan the spatial distribution of the $H_{z}$ component across the field rotator by employing a split-ring antenna. The split-ring antenna has a circular loop with a diameter of $6 \mathrm{~mm}$ which has a $1 \mathrm{~mm}$ split at the front side of the loop, and the back side is broken and connected with the inner and outer conductors of a coaxial cable. The splitring antenna as well as the coaxial cable is mounted on a two-dimensional translation stage and programmed to do point-by-point scanning in the vertical $x-y$ plane. To scan the magnetic field inside the rotator, we cut the field rotator into two segments, each $40 \mathrm{~cm}$ long, and they are put next to each other with their axes aligned. A $5 \mathrm{~mm}$ air gap is maintained between the opposite cross sections of the two

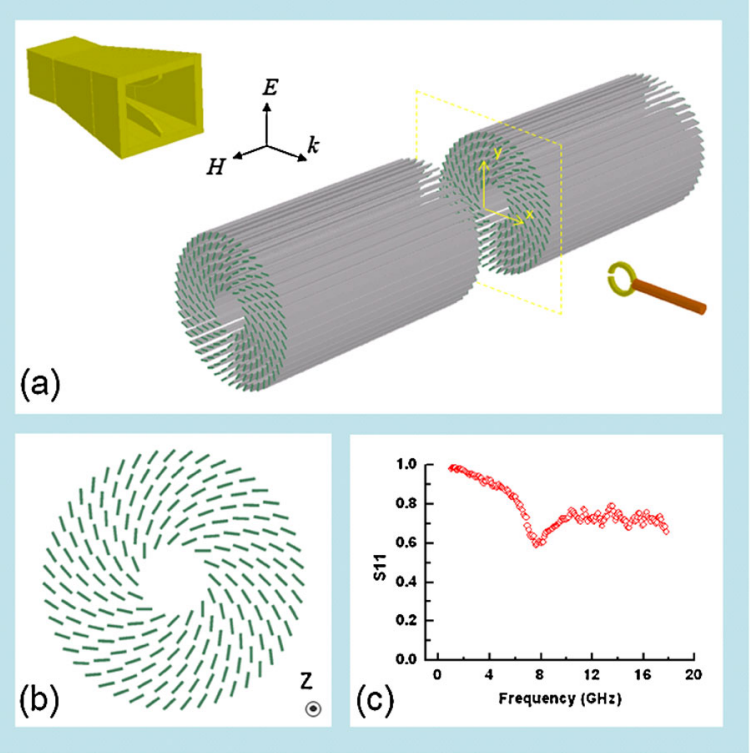

FIG. 2 (color online). (a) A schematic illustration of the samples (two segments of the field rotator) and the measurement setup, where the dashed lines mark the scanning region accessed by the split-ring antenna that is positioned by an automated stepping device. The dark green (dark gray) color on the cross sections of the samples is used to highlight the arrangement of $\mathrm{Al}$ pieces in the field rotator. The air gap between two segments of the field rotator has been exaggerated for visualization. (b) The arrangement pattern of the $\mathrm{Al}$ pieces viewed from the $+z$ direction. (c) The $S 11$ response of the split-ring antenna.

segments to allow penetration of the split-ring antenna, as illustrated in Fig. 2(a). The scanning plane that is parallel to the two cross sections of the two segments of the field rotator is adjusted to pass through the air gap. The scanning area covers an area of $15 \times 15 \mathrm{~cm}^{2}$ with a spatial resolution of $2 \times 2 \mathrm{~mm}^{2}$, which is marked by the yellow (or light gray) dashed lines in Fig. 2(a). The ring plane of the detector antenna is set to be parallel to the $x-y$ plane to probe the $H_{z}$ component of the magnetic field. The hornshaped antenna and the detector are connected to an $S$-parameter network analyzer to obtain the magnitude and phase spectra of the signal received by the detector [29]. The $S 11$ parameter of the split-ring antenna is measured first and plotted in Fig. 2(c), which shows that magnetic resonance was found at $8.0 \mathrm{GHz}$. Accordingly, we set the sweep frequencies of the network analyzer to center on $8.0 \mathrm{GHz}$ and we record the transmission magnitude and phase spectra, $A(x, y, f)$ and $\varphi(x, y, f)$ (where $f$ is the frequency), respectively, at every spatial point $(x, y)$ sampled by the split-ring antenna.

We utilize an $8.0 \mathrm{GHz}$ TE polarized incident wave that is incident from left to right. The scattering patterns for an ideal and a reduced-field rotator are compared in Figs. 3(a) and 3(b). The inner radius is $r_{1}=12.5 \mathrm{~mm}$ while the outer radius is $r_{2}=50 \mathrm{~mm}$. Figure 3(a) shows the scattering 


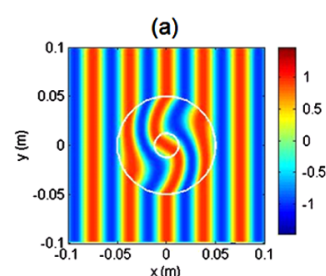

(b)

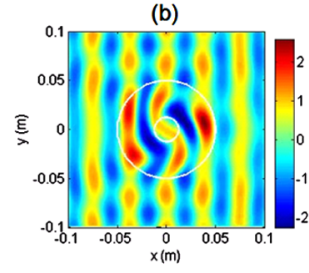

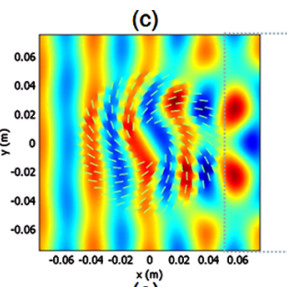

$(\mathrm{e})$

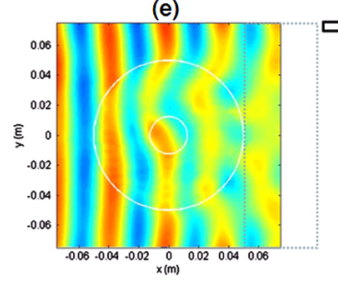

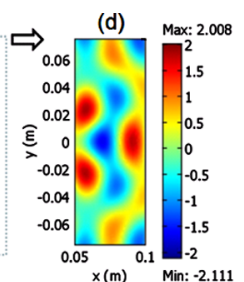

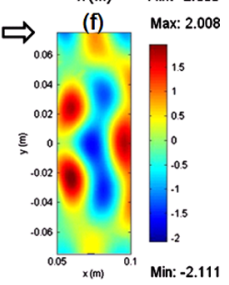

FIG. 3 (color online). (a) The magnetic filed in the vicinity of an ideal field rotator designed by transformation-media theory. The results can be obtained analytically. (b) The magnetic field in the vicinity of a reduced-field rotator (see text). It rotates the field by the same angle as (a), but it causes some scattering. (c) The simulated distribution of the $H_{z}$ component propagating from left to right across the field rotator at $8.0 \mathrm{GHz}$. In this field rotator, the anisotropy is achieved by a collection of rectangular metal plates. It can be viewed as a "discrete" version of the reduced rotator. The shown region measures $15 \times 15 \mathrm{~cm}^{2}$, where the axis unit is in meters. (d) The simulated distribution of the $H_{z}$ component immediately behind the field rotator $(x>$ $50 \mathrm{~mm}$ ) at $8.0 \mathrm{GHz}$. (e) The measured distribution of the $H_{z}$ component across the air gap depicted in Fig. 2(a) at $8.0 \mathrm{GHz}$. The scanning region covers $15 \times 15 \mathrm{~cm}^{2}$, and the white circles outline the interior and exterior boundaries of the field rotator. (f) The measured distribution of the $H_{z}$ component immediately behind the middle part of a segment of the field rotator $(x>$ $50 \mathrm{~mm}$ ) at $8.0 \mathrm{GHz}$. Plots (c) and (d) share a color bar, and plots (e) and (f) share a color bar.

pattern of a ideal field rotator with $\varepsilon_{u}=0.5, \varepsilon_{v}=2, \mu_{z}=$ 1 , and $\tau=\arccos (1 / 3)$. Figure 3(b) shows the scattering pattern of a reduced-field rotator with $\varepsilon_{u}=1.25, \varepsilon_{v}=5$, $\mu_{z}=0.4$, and $\tau=\arccos (1 / 3)$. As discussed previously, both can do field rotation, and the ideal rotator itself causes no scattering while the reduced-field rotator causes some scattering. Figures 3(c) and 3(d) show the simulated distribution of the $H_{z}$ component propagating from left to right across the structure at $8.0 \mathrm{GHz}$. Figure $3(\mathrm{e})$ shows the corresponding experimental result of the distribution of $\mathrm{H}_{z}$ at $8.0 \mathrm{GHz}$, measured as described above. We obtained the real part of the measured quantity from the relation $A(x, y, f) \cos [\varphi(x, y, f)]$ to compare the results with the simulation. The white circles in Fig. 3(e) outline the interior and exterior boundaries of the field rotator, and the slightly curved wave front of the incident plane wave is due to the finite distance between the samples and the source (the horn-shaped antenna). We see that at the center region a $\sim 30^{\circ}$ rotation of the electromagnetic wave occurs with respect to the waves lying outside the field rotator. The agreement with the simulation is very good, except for a decrease in the intensity of the $H_{z}$ component across the field-rotator body with the $x$ coordinate. This is caused by the air gap in the measurement configuration, as mandated by the experimental setup. An $8.0 \mathrm{GHz}$ wave can only tunnel through a $5 \mathrm{~mm}$ wide air gap (which has a cutoff frequency of about $30 \mathrm{GHz}$ to vertically polarized electromagnetic waves), which leads to a decrease in the intensity of the electromagnetic field inside the field-rotator body as well as behind the field rotator. We also ran a scan immediately behind the middle part of a segment of the field rotator far from the air gap [see Fig. 3(f)] and the result behind the field rotator $(x>50 \mathrm{~mm})$ shows full agreement with the simulation [see Fig. 3(d)]. This agreement demonstrates that the simulation can be trusted to a high degree of confidence, and the experimental realization also faithfully produced the desired effect. The major reason for the difference in the transmitted intensity between the measured and simulated field rotation is caused by the cutting of the metamaterial cylinder into two segments for the insertion of the automated step field sensor.

To study the bandwidth of the rotation effect, we also used a small dipole antenna to detect the rotation effect over a frequency band at three selected positions: $P 1$ $(5.8 \mathrm{~cm}$ in front of the field rotator), $P 2$ (on the central axis inside the field rotator), and $P 3(2 \mathrm{~cm}$ behind the field rotator), which are shown in Fig. 4(a). The $15 \mathrm{~mm}$ long dipole antenna was positioned at these points and was rotated in the $x-y$ plane. The transmission magnitude spectra were measured at different $\theta$ (denoting the angle between the dipole and the vertical direction) at these three positions. The results are presented in Figs. 4(b)-4(d). We

(a)
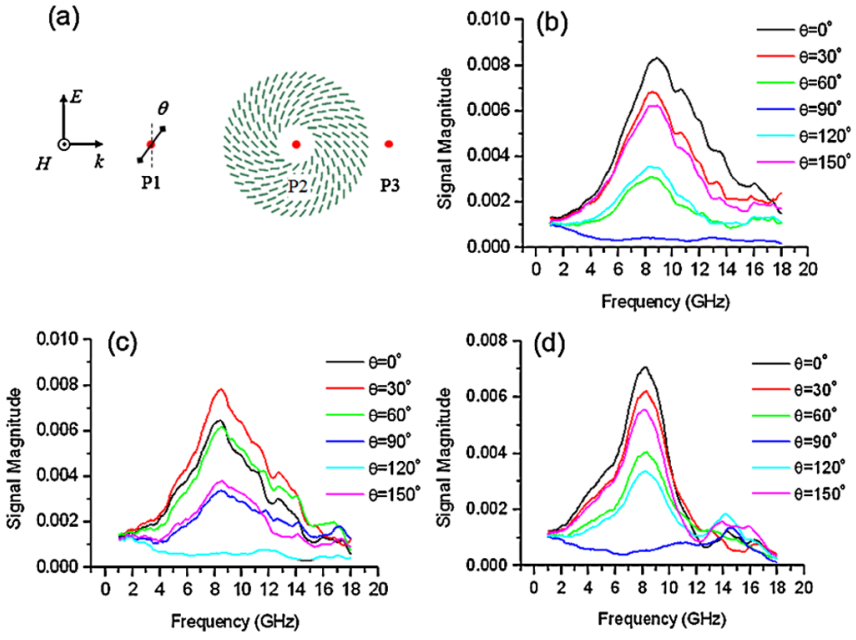

FIG. 4 (color online). (a) A schematic of three selected positions to detect the rotation effect of the electromagnetic field. The wave is incident from the left side. $P 1$ is $5.8 \mathrm{~cm}$ in front of the field rotator; $P 2$ is on the central axis inside the field rotator; and $P 3$ is $2 \mathrm{~cm}$ behind the field rotator. $\theta$ indicates the angle between the dipole (solid line) and the vertical direction (dashed line). (b), (c) and (d) are the responses of the dipole antenna at the three positions, $P 1, P 2$, and $P 3$, respectively. 
see that the signal magnitude is maximal at $\theta=0^{\circ}$ and minimal at $\theta=90^{\circ}$ both in front of and behind the field rotator [see Figs. 4(b) and 4(d)]. However, on the central axis inside the field rotator, the signal magnitude is maximal when $\theta=30^{\circ}$ and minimal when $\theta=120^{\circ}$ [see Fig. 4(c)], which verifies the $30^{\circ}$ rotation of polarization inside the field rotator over a wide frequency band ranging from 2 to $10 \mathrm{GHz}$.

In conclusion, we have designed a field rotator using transformation optics. A reduced form of the rotator has a very simple geometry. We fabricated a rotator based on our design and its field rotation functionality was demonstrated in microwave experiments. We found rotation effect predicted theoretically in a broad frequency range.

This work was supported by a Hong Kong Research Grants Council Central Allocation grant (HKUST3/06C). The computation resources were supported by the Shun Hing Education and Charity Fund.

*phchan@ust.hk

[1] U. Leonhardt, Science 312, 1777 (2006).

[2] J. B. Pendry, D. Schurig, and D. R. Smith, Science 312, 1780 (2006).

[3] D. Schurig, J. J. Mock, B. J. Justice, S. A. Cummer, J. B. Pendry, A. F. Starr, and D. R. Smith, Science 314, 977 (2006).

[4] D. Schurig, J. B. Pendry, and D. R. Smith, Opt. Express 14, 9794 (2006).

[5] W. Cai, U. K. Chettiar, A. V. Kildishev, and V. M. Shalaev, Nat. Photon. 1, 224 (2007).

[6] H. Y. Chen, Z. Liang, P. Yao, X. Jiang, H. Ma, and C. T. Chan, Phys. Rev. B 76, 241104(R) (2007).

[7] H. Chen, B.-I. Wu, B. Zhang, and J. A. Kong, Phys. Rev. Lett. 99, 063903 (2007).

[8] Z. Ruan, M. Yan, C. W. Neff, and M. Qiu, Phys. Rev. Lett. 99, 113903 (2007).

[9] W. Cai, U. K. Chettiar, A. V. Kildishev, V. M. Shalaev, and G. W. Milton, Appl. Phys. Lett. 91, 111105 (2007).

[10] J. Li and J.B. Pendry, Phys. Rev. Lett. 101, 203901 (2008).
[11] U. Leonhardt and T. Tyc, Science 323, 110 (2009).

[12] R. Liu, C. Ji, J. J. Mock, J. Y. Chin, T. J. Cui, and D. R. Smith, Science 323, 366 (2009).

[13] M. Rahm, D. Schurig, D. A. Roberts, S. A. Cummer, D. R. Smith, and J. B. Pendry, Photon. Nanostr. Fundam. Appl. 6, 87 (2008).

[14] H. Y. Chen and C. T. Chan, Appl. Phys. Lett. 90, 241105 (2007).

[15] Y. Luo, H. S. Chen, J. Zhang, L. Ran, and J. A. Kong, Phys. Rev. B 77, 125127 (2008).

[16] A. Greenleaf, Y. Kurylev, M. Lassas, and G. Uhlmann, Phys. Rev. Lett. 99, 183901 (2007).

[17] A. V. Kildishev and E. E. Narimanov, Opt. Lett. 32, 3432 (2007).

[18] M. Rahm, S. A. Cummer, D. Schurig, J. B. Pendry, and D. R. Smith, Phys. Rev. Lett. 100, 063903 (2008).

[19] H. Y. Chen and C. T. Chan, Phys. Rev. B 78, 054204 (2008).

[20] H. Y. Chen, X. Luo, H. Ma, and C. T. Chan, Opt. Express 16, 14603 (2008).

[21] M. Yan, W. Yan, and M. Qiu, Phys. Rev. B 78, 125113 (2008).

[22] T. Yang, H. Y. Chen, X. Luo, and H. Ma, Opt. Express 16, 18545 (2008).

[23] J. Ng, H. Y. Chen, and C. T. Chan, Opt. Lett. 34, 644 (2009).

[24] Y. Lai, H. Y. Chen, Z. Q. Zhang, and C. T. Chan, Phys. Rev. Lett. 102, 093901 (2009).

[25] J. Yao, Z. Liu, Y. Liu, Y. Wang, C. Sun, G. Bartal, A. Stacy, and X. Zhang, Science 321, 930 (2008).

[26] J. Valentine, S. Zhang, T. Zentgraf, E. Ulin-Avila, D. A. Genov, G. Bartal, and X. Zhang, Nature (London) 455, 376 (2008).

[27] X. Hu, C. T. Chan, J. Zi, M. Li, and K.-M. Ho, Phys. Rev. Lett. 96, 223901 (2006).

[28] See EPAPS Document No. E-PRLTAO-102-035921 for a specific design using the effective medium theory. For more information on EPAPS, see http://www.aip.org/ pubservs/epaps.html. For the effective medium theory, see D. R. Smith, S. Schultz, P. Markos, and C.M. Soukoulis, Phys. Rev. B 65, 195104 (2002).

[29] B. J. Justice, J. J. Mock, L. Guo, A. Degiron, D. Schurig, and D. R. Smith, Opt. Express 14, 8694 (2006). 